\title{
Variation of presence/absence genes among Arabidopsis populations
}

\author{
Shengjun Tan ${ }^{\dagger}$, Yan Zhong ${ }^{\dagger}$, Huan Hou, Sihai Yang ${ }^{*}$ and Dacheng Tian ${ }^{*}$
}

\begin{abstract}
Background: Gene presence/absence (P/A) polymorphisms are commonly observed in plants and are important in individual adaptation and species differentiation. Detecting their abundance, distribution and variation among individuals would help to understand the role played by these polymorphisms in a given species. The recently sequenced 80 Arabidopsis genomes provide an opportunity to address these questions.

Results: By systematically investigating these accessions, we identified 2,407 P/A genes (or 8.9\%) absent in one or more genomes, averaging 444 absent genes per accession. 50.6\% of P/A genes belonged to multi-copy gene families, or $31.0 \%$ to clustered genes. However, the highest proportion of P/A genes, outnumbered in singleton genes, was observed in the regions near centromeres. In addition, a significant correlation was observed between the P/A gene frequency among the 80 accessions and the diversity level at P/A loci. Furthermore, the proportion of P/A genes was different among functional gene categories. Finally, a P/A gene tree showed a diversified population structure in the worldwide Arabidopsis accessions.
\end{abstract}

Conclusions: An estimate of P/A genes and their frequency distribution in the worldwide Arabidopsis accessions was obtained. Our results suggest that there are diverse mechanisms to generate or maintain P/A genes, by which individuals and functionally different genes can selectively maintain P/A polymorphisms for a specific adaptation.

\section{Background}

Sequence variants affecting phenotypes between different individuals were believed to be mostly due to small differences, such as single nucleotide polymorphisms (SNPs) [1-4]. However, when comparing two or more genomes within a species, gene presence/absence (P/A) variations have been also commonly observed in recent studies. Since Grant et al. found P/A polymorphisms in the RPM1 gene in Arabidopsis [5], an increasing number of P/A genes have been reported in disease resistance genes in this model species [6,7] and other land plants [8-10]. This phenomenon has also been described in the human genome [11-13], the telomeric region in Drosophila [14] as well as bacterial genomes [15], which suggests that P/A polymorphisms have unique roles in species differentiation. Additionally, several human diseases have been associated with gene insertions or deletions $[16,17]$ and in plants, there is evidence that $\mathrm{P} / \mathrm{A}$ genes are involved in

\footnotetext{
* Correspondence: sihaiyang@nju.edu.cn; dtian@nju.edu.cn

${ }^{\dagger}$ Equal contributors

State Key Laboratory of Pharmaceutical Biotechnology, Department of Biology, Nanjing University, Nanjing 210093, China
}

gene expression [18] and noncollinearity in heterosis [19]. These examples indicate the importance of P/A genes in the evolutionary history of various species.

The commonly used definition of a P/A gene is that it is a gene present in some individuals but absent in others within a species at a particular locus, although there are different definitions in the literature $[6,10]$. The narrow definition of a P/A gene is one which exists only in one individual but not in another on a genomewide scale. For example, it was reported in maize that $20 \%$ of genome segments $(\sim 10,000$ genes or gene fragments) are not shared between inbred lines B73 and Mo17 [8]. Yu et al. found that $2.2 \%$ and $3.3 \%$ of rice indica and japonica genes, respectively, are unique to the subspecies [20], while Ding et al. found 5.2\% genes with P/A polymorphisms between Nipponbare and 9311 [10]. Although a gene can be localized to a genomic position and be denoted as a P/A gene at that locus, it may have a paralog at a different locus. By using a broad definition, $4.7 \%$ additional genes were classified as P/A genes among rice genomes [10]. Our study also uses the broad definition of a P/A gene, which is one being found 
at a particular locus only in some genomes compared to the others.

Most land plants have evolved by whole genome duplication and subsequent gene loss [21]. Such extensive rearrangement events can result in a high proportion of P/A genes in plants. Transposable elements (TE) are dominant factors inducing intraspecies diversity in maize [8]. Large duplications can be another source of genetic variation [22]. In Arabidopsis, unequal and illegitimate recombination also plays an important role in triggering large-scale indels [23]. The Arabidopsis genome is extremely redundant due to segmental duplications and tandem arrays [24]. These features provide ample opportunity for unequal crossing over to generate P/A genes. Balancing selection is thought to be one of the mechanisms maintaining $\mathrm{P} / \mathrm{A}$ polymorphisms, at least for some disease resistance P/A genes $[6,7,25]$. However, compared with the large numbers of detected P/A polymorphisms, the mechanisms for $\mathrm{P} / \mathrm{A}$ gene generation and maintenance are complicated and remain unclear.

Although P/A polymorphisms have been reported in several species $[6,23]$, there is still a lack of a clear estimate of the P/A gene number, proportion and variation pattern in any particular species, since a large number of fully sequenced individual genomes is the basic prerequisite for such studies. Recently, 80 re-sequenced Arabidopsis genomes were released [26,27] and provided a unique opportunity to systematically study the characteristics of P/A genes. By analyzing the data, we identified a remarkable number of P/A genes and obtained an estimate of the P/A genes and their frequency distribution in the worldwide Arabidopsis accessions. We also used this information to investigate the variation in P/A gene patterns among accessions and to provide a description of their preference locations on chromosomes. An analysis of the relationship between diversity and frequency of $\mathrm{P} / \mathrm{A}$ genes was performed to explore the natural selection pressure, the evolutionary forces on P/A genes in Arabidopsis populations as well as the mechanism for $\mathrm{P} / \mathrm{A}$ generation.

\section{Results}

\section{Identification of P/A genes}

Out of the total 27,004 genes in the Arabidopsis thaliana Columbia (Col) line, 2741 genes (10\%) were found absent in one or more genomes of the 80 Arabidopsis accessions using our approach. Among these genes, 603 were absent in only one accession as shown in Figure 1A. As the absent allele frequency in accessions increased, the number of missing genes decreased rapidly, except at the last outlying datapoint on this graph (Figure 1A) which represents 334 genes absent in all 80 accessions. In other words, these genes were present only in the Col genome.
Since a remarkable proportion (10\%) of genes were found absent in multiple Arabidopsis accessions, it was especially important to determine whether the identified genes were truly $\mathrm{P} / \mathrm{A}$ loci. Three methods were used to evaluate the reliability of the identification of these genes. First, we built a random model to predict the number of absent genes for a certain number of accessions by using data from the identified P/A genes in the 80 accessions. In this manner, a formula $(y=480.91 n$ $(\mathrm{x})+252.6)$ was obtained when up to 80 accessions were randomly sampled (Figure 1B). The trendline in Figure $1 \mathrm{~B}$ demonstrates that the number of absent genes increases logarithmically when the number of accessions sampled increases linearly, and we could estimate the absent gene number at each datapoint. For example, when 4 accessions are sampled, a total of 919 absent genes are expected based on the formula. Fortunately, there are four independent populations (C24, Bur-0, Kro-0 and Ler-1; [28]) which have been fully sequenced with high genome coverage $(50-200 \times)$. We used the same methods as for the 80 accessions to identify the $\mathrm{P} /$ A genes in these four populations. A consistent result between the number of absent genes identified from independent samples and that predicted by the formula above would help to validate the P/A genes identified in the 80 accessions. In fact, $980 \mathrm{P} / \mathrm{A}$ genes were identified in the four independent populations, not significantly deviated from the number (919) predicted by the formula above (from randomization: $P=0.77$ ), indicating a general reliability of the P/A genes identified.

Second, we determined whether some of the P/A genes were truly absent by PCR. We randomly chose 53 genes for genotyping in randomly selected accessions. Of the 38 less frequent P/A genes which were absent in one or ten accessions, nine genes (or 23.7\%) were determined to be false (Additional file 1: Table S2; Additional file 2: Table S4). Meanwhile among the remaining 15 genes, which had a higher absence frequency, two of them (13.3\%) were not true P/A genes. Therefore, 11 out of the $53 \mathrm{P} / \mathrm{A}$ genes were not truly absent, while the other 42 were confirmed to be absent (33 out of 42) or did not generate PCR products (9 out of 42), most likely due to the absence of the regions containing the primer sequences. Our PCR results demonstrated that the rate of wrongly inferred P/A genes was $25 \%(=11 /(53-9))$ after excluding the uncertain results, while the majority of P/A genes (75\%) initially identified were truly absent.

Finally, we evaluated the reliability of the outlier in Figure $1 \mathrm{~A}$, the last datapoint with the abnormally high value of $334 \mathrm{P} / \mathrm{A}$ genes. This number could be accurate when there are large numbers of population-specific genes. In this case, it was reasonable for Col, which was supported by BAC-clone based genome sequences, to have many P/A genes; however, the other accessions 

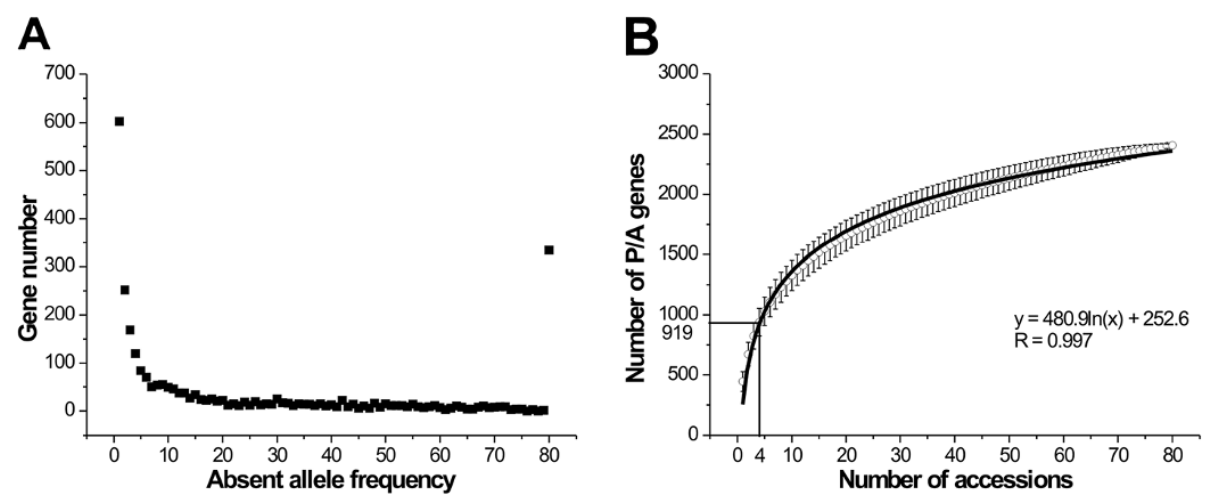

Figure 1 Distribution of P/A frequencies among 80 Arabidopsis accessions. (A) Number of P/A genes vs. absent allele frequency. (B) Expected increase of P/A genes relative to the number of sampled accessions, based on a random sampling model. Each datapoint represents an average number of P/A genes for 10000 random simulation samples, and the 95\% confidence intervals were also indicated. For example, when 4 accessions are sampled, 919 absent genes are expected.

could not have had such high numbers, because the resequenced genomes used $\mathrm{Col}$ as a reference and therefore would not produce additional accession-specific P/ A genes. However, when all 334 genes were subjected to BLAST search against the four genomes of C24, Bur-0, Kro-0 and Ler-1, 198 out of the 334 genes produced no matches, while 8 had alleles in all four genomes. The BLAST results suggested that there were accessionspecific P/A genes, but a large proportion (40.7\%) of the 334 genes were not reliable, probably because these genes could not be easily re-sequenced in the 80 accessions. Thus, all of the genes at this datapoint were discarded in our subsequent analyses.

Of the remaining 2,407 P/A genes, the average number of absent genes per accession was 444 (ranging from 362 to 555 ), which meant that $1.64 \%$ of the genes were absent compared with those of Col. This proportion was slightly lower than that reported in rice $[10,20]$ and maize [8]. Additionally, nine Arabidopsis resistance genes with known P/A polymorphisms [6] were found to be included in these P/A genes, further supporting the validity of the method for their identification in this study.

\section{Characteristics of P/A genes among populations}

We next determined whether the P/A genes were transcriptionally active. Some genes, such as pseudogenes are not expressed, and therefore they would not affect biological processes in an individual. After BLAST searches in the Arabidopsis Transcriptome Genomic Express Database, we found evidence of expression for all of the 2,407 genes, suggesting that the P/A genes have biological functions. However, this analysis was performed based on the Col annotation, and we could not confirm expression of these genes from other accessions.

It is rational to assume that many $\mathrm{P} / \mathrm{A}$ genes may come from multi-copy gene families, and the homologous genes may have overlapping functions. To better group the homologs, genes with a nucleotide divergence less than 0.3 were defined as a family. By this definition, 1,218 P/A genes (or 50.6\%) were grouped into 718 families, but 1,189 singleton genes (or 49.4\%) still lacked homologs in the Col genome. In the 24,570 non-P/A genes $(27,004$ total genes minus the 2,407 P/A genes), there were 8,247 genes (33.5\%) which belonged to multi-gene families. There was a significantly higher proportion of multi-genes in P/As than that of non-P/A genes $\left(\chi^{2}=281, \mathrm{df}=1\right)$, implying that the multicopy genes were more likely to have $\mathrm{P} / \mathrm{A}$ polymorphisms.

Large deletions or the absence of genes can be derived from unequal recombination between homologs [23]. Tandemly arranged or clustered genes are also abundant resources for generating gene conversion and unequal crossover [29]. Therefore, the P/A genes may be a consequence of unequal recombination between clustered genes. Here, we classified the P/A genes into clusters to investigate their physical locations (definitions in Methods). In total, 1,272 gene clusters were found in the $\mathrm{Col}$ genome, of which 463 clusters contained 746 P/A genes. In other words, $31.0 \% \mathrm{P} / \mathrm{A}$ genes were clustered on chromosomes, significantly higher than the $12.0 \%$ of clustered genes in the whole Col genome $\left(\chi^{2}=915\right.$, $\mathrm{df}=1)$.

\section{Co-variation between absent allele frequency and nucleotide diversity or gene length}

The average nucleotide diversity of all the present alleles at the P/A loci was 0.0088 . The level of nucleotide diversity may be associated with the frequency of absent alleles within a population. According to this hypothesis, a high frequency of absent alleles could be maintained with higher probability by balancing selection, an indication of higher diversity at a locus. To detect this association, the 2,407 P/A genes were equally sorted into 29 
bins based on their frequencies among accessions, such that each of these bins contained 83 genes. Figure 2A shows the relationship between the frequency of absent alleles and the diversity of present alleles at a P/A locus. The increase in absent allele frequency (represented by the number of absent alleles from 1 to 80 accessions) positively correlated with the average nucleotide diversity until the number of absent alleles reached 40 out of 80 accessions (or $\sim 0.5$ frequency of absent alleles), after which the average diversity decreased. Thus, there was a significant quadratic-like correlation between the nucleotide diversity and frequency of absent alleles $(\mathrm{r}=0.65 ; P<0.0001)$. Additionally, a similar pattern was found when investigating the relationship between the frequency of absent alleles and the length of P/A genes (Figure 2B). The implications of these correlations remain to be clarified.

\section{Variation of P/A genes among populations}

To examine variations in the pattern of $\mathrm{P} / \mathrm{A}$ genes among accessions, a phylogenetic tree, based on the presence or absence of the 2,407 genes, was constructed. We observed some extent of differentiation in these genes among the 80 accessions based on geographic origin (Additional file 3: Figure S5A). Many genes with similar polymorphic patterns were found in accessions from the same countries. That is, these closely located accessions were likely to have similar spectrums of P/A genes. Some accessions from the same geographic area, such as Bak-2 and Bak-7, had highly similar patterns of $\mathrm{P} / \mathrm{A}$ genes.

To better characterize the geographic distribution of $\mathrm{P} / \mathrm{A}$ polymorphisms, a reconciled tree (Figure 3) was constructed to view the evolved P/A tree over an accession tree, built by using all SNPs of non-P/A genes (Additional file 3: Figure S5B). In the reconciled tree, we could see that the 80 accessions (shown in blue) were generally separated into eight geographic regions where they were sampled, and the P/A tree (shown in black) matched the accession tree particularly in each of the 8 clades (Figure 3), indicating there was a strong correlation between P/A pattern and individual variation. Both trees (reconciled and P/A trees) demonstrated that P/A polymorphisms were related to the geographic regions, suggesting that population structure may play an important role in the generation/maintenance of $\mathrm{P} / \mathrm{A}$ genes.

However, each branch (or accession) was quite different from others in the P/A gene tree. It was especially remarkable that no identical branches could be detected among the 80 accessions. The closest accessions were ICE169 and ICE173 (Additional file 4: Table S1), which had no SNP detected in the non-P/A genes between them (Additional file 3: Figure S5A), but they still had different $\mathrm{P} / \mathrm{A}$ polymorphic patterns at 26 loci. The same phenomena were found in comparisons between ICE150 and ICE152 as well as between ICE212 and ICE 213.

\section{Distribution of $\mathrm{P} / \mathrm{A}$ genes on chromosomes}

The five chromosomes were divided into $1 \mathrm{Mb}$ sections and centromere positions were established as previously reported [30]. Interestingly, a markedly nonrandom distribution of P/A genes was revealed along the chromosomes (Figure 4). The proportion of P/A genes (the number of P/A genes divided by number of all genes in each section) was always the highest near the centromere, and decreased monotonically to background levels over several megabases. This phenomenon could be attributed to the low gene density in the areas around centromeres, and thus the gene-rich regions were expected to have more P/A genes but not higher proportions. However, detailed examination showed the opposite finding. For example on chromosome 3, the highest gene-density section with a total of 329 genes contained only nine P/A genes. Meanwhile there were $27 \mathrm{P} / \mathrm{A}$ genes in the centromeric section where there were a total of 33 genes. The same pattern was also observed on chromosome 5 (5:307 and 12:18, respectively, for P/A to total genes) and other chromosomes (Additional file 5: Figure S1; Additional file 6: Table S3).
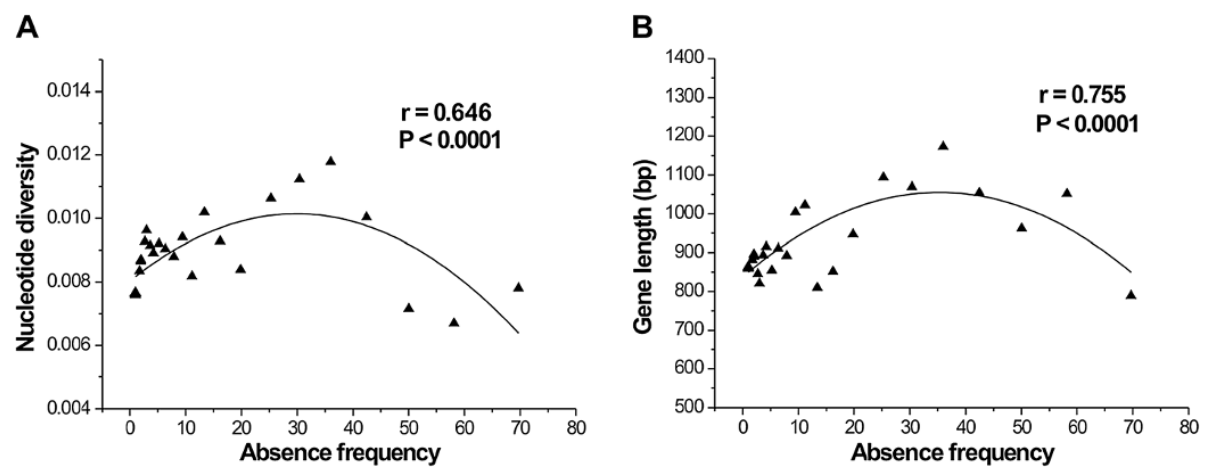

Figure 2 Co-variation between absent allele frequency and nucleotide diversity (A) or gene length (B) 


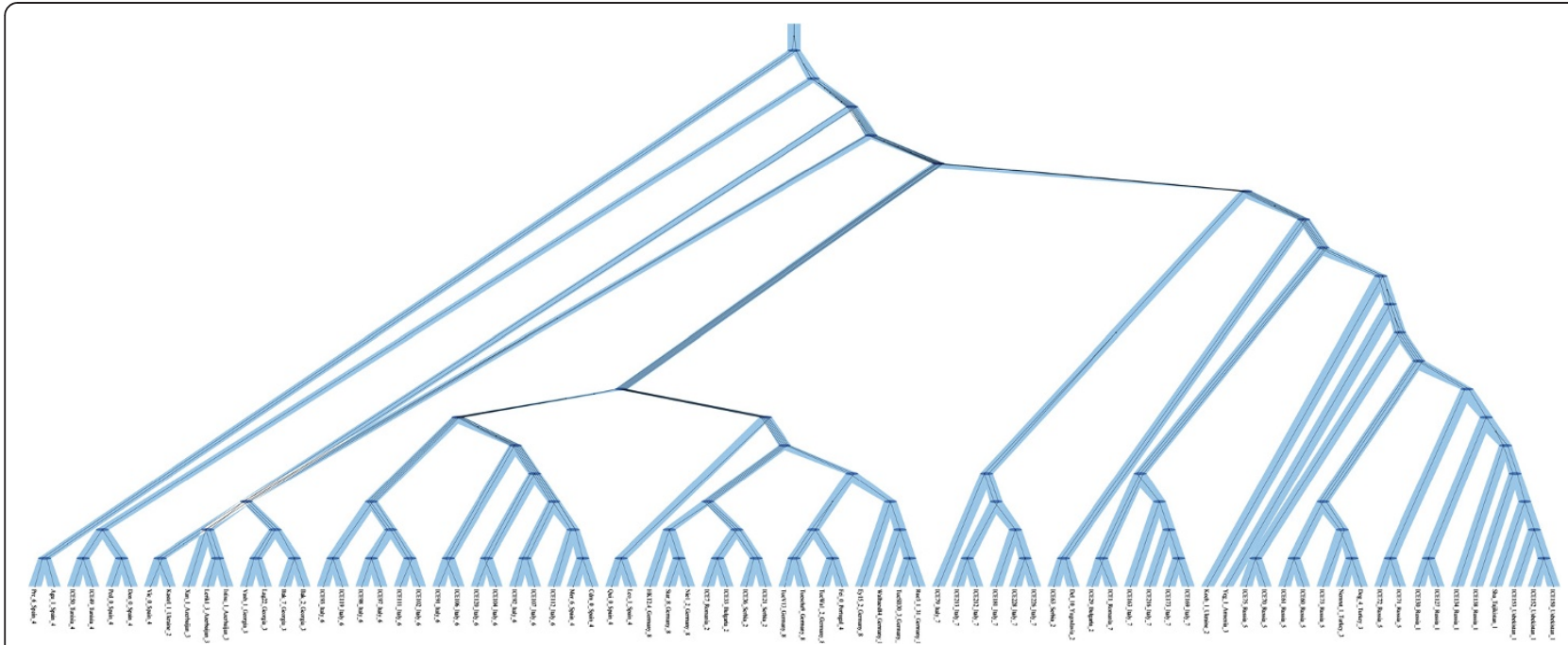

Figure 3 The reconciled tree. This tree was viewed as the evolved P/A tree inside the accession tree. The accession tree was shown in blue and the P/A tree in black. At the edges of branches were the accession names, countries and 8 regions where they were sampled. Most of the P/A tree vertices matched the accession tree vertices (dark blue ellipses) particularly in each geographic clade, which meant the P/A polymorphic pattern was associated with individual variation.

\section{Functional categories of P/A genes}

Gene Ontology (GO) terms are widely used as the standard for functional annotation. The 22,570 Arabidopsis genes have been assigned at least one GO annotation from the TAIR and The Institute of Genomic Research (TIGR) databases [31]. The three organizing principles of GO are cellular component, molecular function and biological process [32], and according to the 'GO Slim' categories, genes are classified into small groups within each GO hierarchy.

Of the cellular component genes, $738 \mathrm{P} / \mathrm{A}$ genes (65.9\%) were related to membranes, comprising the largest proportion besides unknown components (Additional file 7: Figure S3). Compared to the overall frequency of membrane genes $(37.5 \%,[31])$, the membrane-related P/A genes were significantly overrepresented $\left(\chi^{2}=359, \mathrm{df}=1\right)$. Most of them encoded proteins in the endomembrane system (555 $\mathrm{P} / \mathrm{As}$ ), and the others were distributed within the plasma, chloroplast and Golgi membranes. When analyzing the molecular functions, 888 P/A genes (48.8\%) encoded proteins with binding functions, mostly binding with protein and nucleotides, and were also overrepresented $\left(\chi^{2}=167\right.$, $\mathrm{df}=1$ ). However, 121 encoded proteins in the 'other binding' category could bind to zinc ion, while the remaining proteins had various ion binding functions. The activity terms of many enzymes, including hydrolases, transferases and kinases, were also numerous in the molecular function category. In the category of biological process, the highest proportion of these were related to stress responses $(23.8 \%$ vs $2.2 \%, \chi^{2}=1466, \mathrm{df}=1$ ).

Moreover, we analyzed the frequency of P/A genes in different gene classes identified by Pfam domain database [33]. In Arabidopsis, 75\% 82\% functional domains could be predicted by Pfam [34,35], and such a high proportion could cover most of the fundamental gene classes. Classes involved in basic biological processes (such as heat shock protein and ABC transporter) and in regulation of transcription (such as Myb and HLH) had less impact (see Figure 5). By contrast, disease resistance genes and F-box gene classes were affected extensively ( $53.9 \%$ and $16.6 \%$, respectively). In NBS proteins, the LRR domains are thought to mediate interactions with pathogen-derived molecules and reported to be highly variable [36]. This finding is consistent with previous studies in which the NBS genes often show P/A polymorphisms in Arabidopsis and other plants $[5,6,10,37]$. Additionally, F-box genes are thought to have undergone rapid birth and death in the Arabidopsis genome [38], as well as evolved quickly in response to pathogen pressure [39].

\section{Discussion}

\section{Number of P/A genes among populations}

Using our approach, 2,407 genes were identified with P/ A polymorphisms, indicating that about $10 \%$ of genes are likely to be maintained as P/A genes in Arabidopsis. Both the number and the proportion of $\mathrm{P} / \mathrm{A}$ genes are remarkable, which may reflect their important roles in the species adaptation to the environment.

However, our approach could have overestimated the number of P/A genes. The PCR results indicated that 25\% of the P/A genes were falsely identified. On the other hand, the number identified could be underestimated, because the proportion of false P/A genes was predicted to be much 

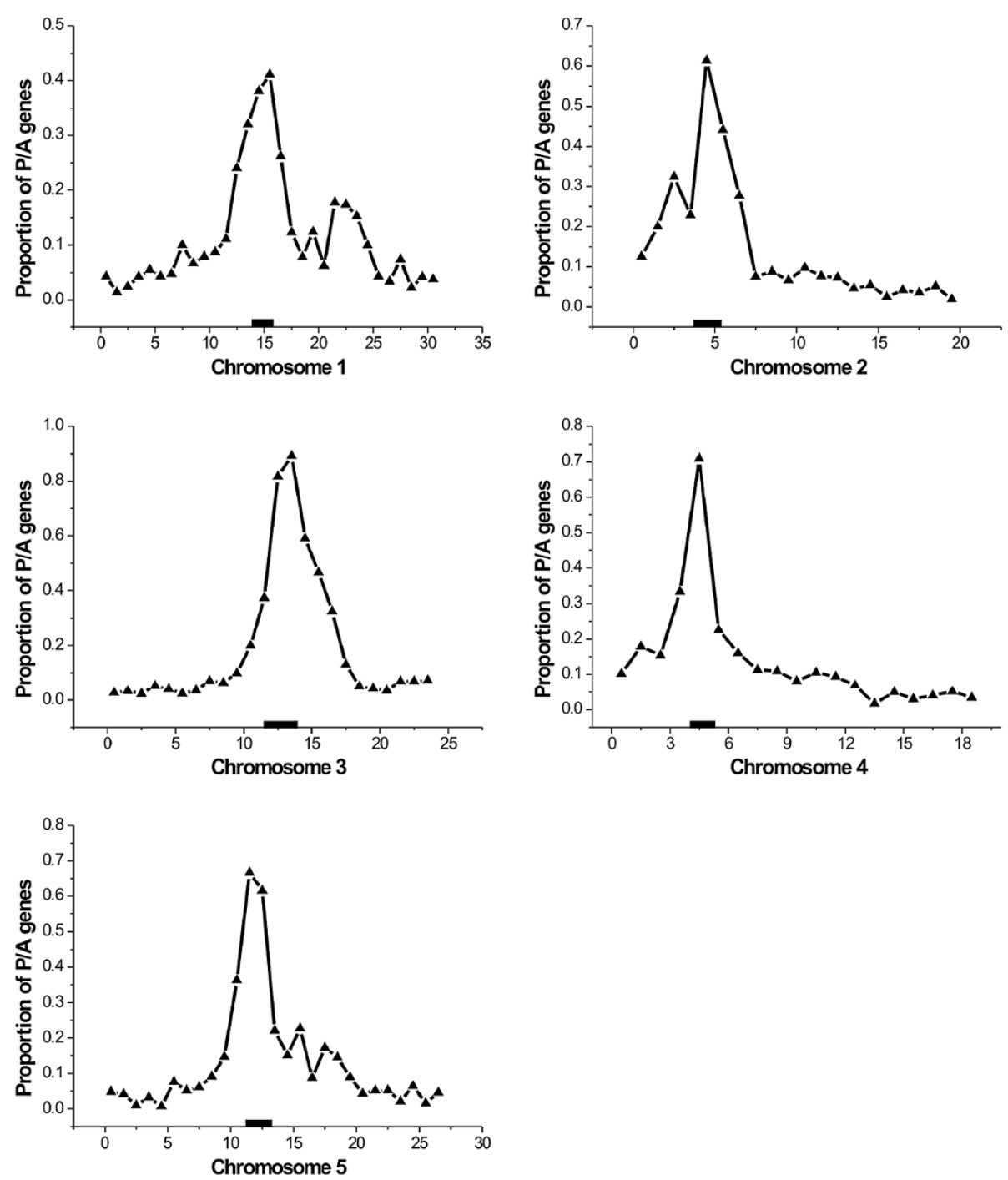

Figure 4 Distributions of P/A genes on chromosomes. The $x$-axis represents the physical distance $(\mathrm{Mb})$ on the chromosome. The $y$-axis represents the proportion of P/A genes for each Mb of chromosomal length. The thick black bar represents the centromeric region.

lower when the absent frequency was higher. Moreover, only P/A genes which are in deletions, compared with the Col genome, could be identified, based on the limitation of re-sequencing methods for the 80 genomes. If the $\mathrm{P} / \mathrm{A}$ genes in insertions could be identified, the number of P/A genes could double. In fact, most of the $334 \mathrm{P} / \mathrm{A}$ genes present only in Col but absent in all 80 accessions should represent P/A genes in $\mathrm{Col}$ insertions, relative to the 80 accessions. This number was similar to the average number (444) of P/A genes per accession. In this case, each accession was expected to contain $2.47 \% \mathrm{P} / \mathrm{A}$ genes. Although the expected proportion is likely higher by two-fold, it was lower than that in rice $(5.2 \%)$ and maize $(\sim 20 \%)$ for each individual.

The difference in proportion of $\mathrm{P} / \mathrm{A}$ genes identified here in Arabidopsis, compared with rice and maize, could be due to the genome sizes, methods for pollination, transposon activity and/or domestication. The maize genome undergoes recurrent transposon events; therefore, observation of widespread DNA sequence noncollinearity would not be unexpected [8]. The outcrossing method and the large genome size may also contribute to the high proportion of $\mathrm{P} / \mathrm{A}$ genes. The rice genome is three times larger than that of the A. thaliana genome, which is more compact consisting largely of unique sequences [38]. Moreover, the previous comparison of rice genomes was based on two individual plants under high pressure of artificial selection in different regions [20], which would genetically create higher divergence [40]. These characteristics may explain the greater number of $\mathrm{P} / \mathrm{A}$ genes observed in rice.

Previously, Clark et al. found that 2,495, or 9.4\%, of $A$. thaliana protein coding genes were affected by large 


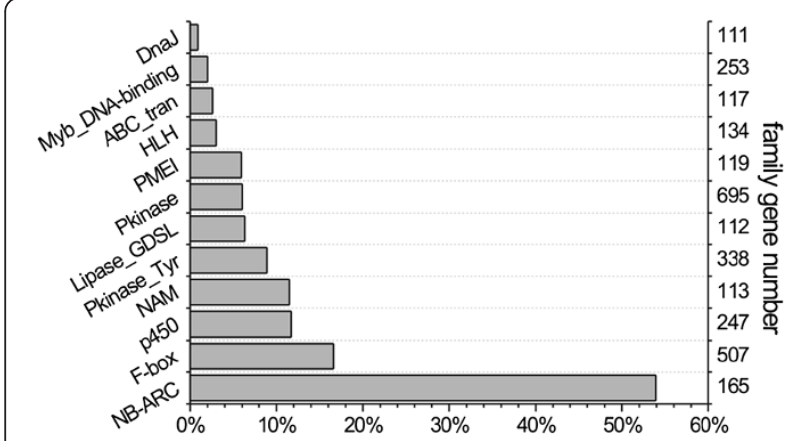

Figure 5 Distribution of P/A genes in different functional

categories. The $y$-axis on the right is the number of genes in each category and the $\mathrm{x}$-axis is the proportion of P/A genes.

effect changes when comparing 19 accessions and one reference, Col [38]. Meanwhile, according to our formula, there should be 1,669 absent genes when 19 accessions were randomly sampled. This number was less than the 2,495 indicated by the authors above because the large effect changes included indels as well as SNPs and highly dissimilar sequences. Ziolkowski et al. found large indels (>100 bp) distributed within 908 CDS regions by comparing genomic data from two $A$. thaliana lines, Col and Ler [23]. This number was also larger than ours since not all indels result in gene deletions.

On the other hand, pseudogenization could be considered as another way of gene loss. Across the 80 accessions, Cao et al. found a total of 6,197 genes with 12,468 SNPs that caused large effects on gene structure, for example altered start codons, introduced premature stop codons, or affected splice donor or acceptor sites, were found [26]. Consistent with their results, we found 7,602 genes contained frameshift mutations or premature stop codons affected by both SNPs and indels in these accessions. The numerous $\mathrm{P} / \mathrm{A}$ and pseudo- genes demonstrated that there were frequent gene loss events in Arabidopsis populations. Most interestingly, P/A and pseudo- genes had similar distribution in gene categories and on chromosomes. For example, there were a high proportion of pseudo- or P/A genes in NBS, F-box and p450 gene families. These results suggested that both pseudo- and P/A polymorphisms may play similar roles in contributing to the dynamic variations in some functional loci, e.g. through pseudogenization and P/A to escape the fitness costs [37].

\section{Mechanisms of P/A gene distribution on chromosomes}

Our results revealed that the distribution of $\mathrm{P} / \mathrm{A}$ genes was not random on chromosomes and that their proportion was especially high around centromeres. The P/A genes could be easily generated by unequal recombination between homologs, especially tandemly arranged genes. In this scenario, a higher proportion was expected in the areas with dense gene clusters. Previous studies $[23,29]$ indeed support this mechanism to engender P/A polymorphisms in A. thaliana. Generally, A. thaliana has a relatively compact genome and contains many unique sequences, e.g., $64.5 \%$ singletons by our definition in which $6.78 \%$ of $\mathrm{P} / \mathrm{A}$ genes were identified. Nevertheless, there was a significantly higher proportion of P/A genes (50.6\%) found in the multi-gene families. Within this multi-gene group with $\mathrm{P} / \mathrm{A}$ genes, a significantly higher percentage of clustered P/A genes (746/ $1,218=61.2 \%)$ was observed. Although with some variation, the proportion of clustered P/A genes was indeed much higher on the chromosome arms (Additional file 8: Figure S2) as expected. Meanwhile on chromosomes 1 and 5, there were several P/A secondary peaks (Figure 4), at which most of the clustered genes were located (Additional file 8: Figure S2; Additional file 6: Table S3).

However, a relatively smaller number of $\mathrm{P} / \mathrm{A}$ genes clustered near the centromeric regions (Additional file 8: Figure S2). This finding suggested that the unequal recombination events among P/A genes were not frequent in these regions, which is also reasonable since centromeres are reported to have low recombination frequencies [41]. However, we observed a high proportion of P/ A genes (the number of $\mathrm{P} / \mathrm{A}$ genes divided by number of all genes) near centromeres, indicating that other mechanisms generated P/A genes in these areas. Centromeres have highly conserved functions, serving as the site for kinetochore formation and sister chromatid cohesion, while it is also the most dynamic chromosomal region $[42,43]$. Although the explanation for rapid evolutionary dynamics is still under debate [44], stochastic events such as mutation and genetic exchange are apparent [45]. Widespread gene conversion has also been observed in these regions [46]. Unlike chromosome arms which have high gene density, the centromere usually contains specific types of DNA sequences that are typically tandem repetitive sequences [41], and its gene density is much lower in higher eukaryotes. Therefore, the characteristics in these areas could induce a high rate of sequence exchanges, which may result in $\mathrm{P} / \mathrm{A}$ polymorphisms. With a similar evolutionary characterization to the centromere, the telomeric region also contains much repetitive sequences. In Drosophila, Kern found a number of duplication and deletion polymorphisms in this region [14]. However, we did not detect a high P/A proportion in Arabidopsis (Figure 4), which could be resulted from the evolutionary and genetic differences between plants and insects.

The generation of $\mathrm{P} / \mathrm{A}$ genes in centromeric regions is clearly different from other areas. In the less repetitive regions, gene clusters could add or delete copies, which eventually could produce P/A genes. Meanwhile in a region with abundant repeats, the unequal crossover 
among repeat sequences could cause the non-clustered genes to be added or lost, particularly singleton genes on chromosome arms and near the centromeres. It seems that there always are efficient ways to engender $\mathrm{P} / \mathrm{A}$ polymorphisms in different regions.

In addition, Arabidopsis underwent a paleopolyploidy event before splitting from Brassica, which could produce abundant homologous genes and repeat sequences. Although gene duplication is the major mechanism of sub- and neo-functionalization for a species to adapt to the new environment [47], the redundant genes are great burdens for a host. Therefore, the gene loss, which includes deletion and pseudogenization, could be an effective strategy to economize the cost. This explanation may primarily account for the higher P/A polymorphisms among homologous genes.

\section{Evolutionary forces for P/A genes in groups and populations}

Based on the GO analysis, the proportion of P/A genes were quite different among gene function categories. Within the GO cellular component group, the proportions of P/A genes related to membrane and binding were found to be much higher than the others, although these two classes of genes are also relatively high within the whole Arabidopsis genome [31]. Most interestingly, in the category of biological process, the 'response to stress' group comprised the highest proportion of $\mathrm{P} / \mathrm{A}$ genes (23.8\%, Additional file 7: Figure S3), while its portion is only $2.4 \%$ of the whole Arabidopsis genome after excluding the biological process unknown genes. In this group, more than half were disease resistance genes. Similar results were observed in the gene class analysis based on Pfam domain database [33] that revealed a high proportion of $\mathrm{P} / \mathrm{A}$ genes in the NBS gene class (53.9\%, Figure 5). The varied distribution of $\mathrm{P} / \mathrm{A}$ genes in different gene groups suggested that their proportions developed under natural selection. Some NBS genes were reported to have P/A polymorphisms as they have hypersensitive responses to an avirulence protein encoded by a pathogen in Arabidopsis [6,37]. The coevolution between the host and pathogen naturally leads to variable characteristics of NBS genes adapting to rapidly changing pathogenic populations. By contrast, classes involved in basic biological processes would contain fewer P/A genes due to their conserved functions.

In this study, an unknown evolutionary force was also indicated in the distribution of P/A genes among accessions, which exhibited little geographic differentiation based on the highly diversified branches in the P/A gene tree (Additional file 3: S5A). The fact that there were no identical branches detected indicated that the $\mathrm{P} / \mathrm{A}$ genes occurred commonly and maintained selectively in the worldwide Arabidopsis populations. For example, the two closest accessions ICE169 and ICE173 (Additional file 4: Table S1) still had different P/A polymorphic patterns at 26 loci (Additional file 3: Figure S5A). Both accessions within this pair were in similar environments, but their P/A patterns were relatively dissimilar. It seems a balance exists to maintain diverse sets of $\mathrm{P} / \mathrm{A}$ genes, beyond the limitation of the geography-specific factors, biotic or abiotic, to enforce the fixation of P/A genes at a location.

\section{Nucleotide diversity of presence alleles at P/A loci}

In our study, we observed a pattern of co-variation between absent allele frequency and nucleotide diversity among populations (Figure 2). To obtain convincing evidence of such a relationship, genes within the same absent frequency were randomly sorted, and then the 2,407 P/A genes were equally sorted into 29 bins of 83 genes each based on their frequencies among accessions. A figure was also plotted based on the average nucleotide diversity under each frequency (Additional file 9: Figure S4A). In these different analyses, a significant covariation was still observed between the values of the nucleotide diversity and their frequencies.

When the absent accession number was about 40, which also meant the P/A frequency was at the highest $(P: A=40: 40)$, the highest average diversity was detected within present alleles. When the P/A frequency was low, the nucleotide diversity was also lower. A high level of diversity at a locus indicates that the polymorphism has been maintained for a longer time. That a higher frequency of a P/A gene equates with an older time of origin suggests that this type of polymorphism is relatively stably maintained, probably by natural selection. Balancing selection may be one of these selective forces, which for example was suggested to act on the RPS5 gene (AT1G12220) [25], with a high absent frequency of $32 / 80$ and a high nucleotide diversity of 0.0136 . Interestingly, the signature of balancing selection at this locus was similar to the patterns observed in this study. This example suggests that balancing selection favors guarding resistance proteins based on the co-evolutionary arms race model [48]. Indeed, when we compared the P/ A gene numbers between different functional categories, $50.6 \%$ of disease resistance associated genes had a relatively high absent frequency (20-60 out of 80 accessions). This proportion was much higher than those in other groups, such as F-box (16.7\%) or p450 (31.0\%). The highest proportions of both P/A genes and absent frequency indicate that some disease resistance related genes are more likely to be under balancing selection to respond to variations in corresponding pathogens. By contrast, a lower diversity means that the gene is relatively young, and therefore its $\mathrm{P} / \mathrm{A}$ frequency is lower and probably subject to random drifting. The younger or 
recently generated P/A genes may be in flux, and their fate may either be to eventually become deleted or fixed by random drifting or natural selection. These types of P/A genes are more likely to be under neutral mutational and population genetic processes.

\section{Conclusions}

We identified a remarkable number of $\mathrm{P} / \mathrm{A}$ genes in the worldwide Arabidopsis populations, which reflected their important roles in individual adaptation. The distribution of P/A genes was uneven on chromosomes and $31.0 \%$ of them were clustered, suggesting that unequal recombination was a major mechanism for generating P/A polymorphisms. However, a high proportion of $\mathrm{P} / \mathrm{A}$ genes were observed near the centromeres, which meant the generation of P/A genes was different in these regions. We also observed a varied distribution of $\mathrm{P} / \mathrm{A}$ genes in different gene groups, which indicated they were under natural selection. Most importantly, little geographic differentiation was found in the distribution of P/A genes among populations, implying that these genes were generated and maintained randomly at relatively high frequency. Additionally, we observed a relationship between diversity and frequency of P/A genes. The high nucleotide diversity and high absent gene frequency suggested that balancing selection could be a mechanism maintaining P/A polymorphisms. This work may help us to better understand the evolutionary forces on P/A genes in Arabidopsis populations as well as the mechanisms for P/A generation.

\section{Methods \\ Databases}

The genome of Arabidopsis ecotype Columbia (Col) was downloaded from The Arabidopsis Information Resource (TAIR) database (http://www.arabidopsis.org/). The sequences of $80 \mathrm{~A}$. thaliana accessions $(10 \sim 24 \times$ coverage) were downloaded from the 1001 Genomes Data Center (http://1001genomes.org; [26]). This data center only provides files describing the position-wise bases of 80 accessions against the Col assembly. Therefore, we assembled the whole genome sequences of each accession according to the TAIR8 annotated positions. Subsequently, the protein coding regions (CDS) of each accession were obtained based on the annotation of TAIR8, while the CDS with ambiguous bases were eliminated. For multi-transcript genes, only the longest CDS was chosen to represent each of those genes. The TE genes were also excluded. All calculations were implemented using Perl scripts.

\section{Identification of absent genes and construction of trees} Some CDS of certain accessions contained inaccessible regions' defined by the 1001 Genomes Data Center. Such inaccessible regions were either large deletions or lacked base calling in re-sequencing analysis. If the length of these regions were more than half of the CDS length, we defined this gene as an absence in this accession. Since some of the absent genes may be caused by sequencing error or misassembly, we evaluated the reliability of the P/A genes by examining the other sequenced genomes. In the data center, there were four assembled $A$. thaliana accessions, C24, Bur-0, Kro-0 and Ler-1 [28]. These four lines were not included in the 80 accessions, and instead were used as reference genomes to validate the $\mathrm{P} / \mathrm{A}$ genes. The predicted genes were examined by BLASTN (e value $<10^{-10}$ ) search in the local database. If one gene had a sequence counterpart which was $>50 \%$ in length, it was determined to be present in this accession.

All of the candidate genes were subjected to BLASTN search for expression analysis. Expression support was given to the 26,541 annotated $A$. thaliana coding genes on the basis of full-length cDNAs, expressed sequence tags (ESTs), massively parallel signature sequencing (MPSS) and genome-wide tiling array transcriptome (http://signal.salk.edu/cgi-bin/atta; [49]).

To group the $A$. thaliana accessions with respect to the pattern of P/A genes, a phylogenetic tree was created by using $\mathrm{P} / \mathrm{A}$ polymorphisms among accessions, based on neighbor-joining method with p-distance model by PAUP* 4.0 [50]. In addition, an accession tree was constructed by using all SNPs of non-P/A genes among genomes, based on the Neighbor-joining method [51]. A reconciled tree, which was viewed as the evolved P/A tree in the accession tree, was then illustrated using primetv software [52].

\section{Classification of multi-copy gene families, gene clusters and functional categories}

We built a local database of all Col genes, and then every gene was subjected to the BLASTN search to identify homologous genes. A criterion of $\geq 70 \%$ nucleotide identity was used to define homologs in multi-copy gene families [10]. Otherwise, genes were categorized as singletons.

A gene cluster was defined as a region that contained two or more homologs which were close to each other ( $\leq$ eight genes between any two of them, [53]). All absent genes were searched whether they resided together in a gene cluster or not. Subsequently, these genes were subjected to searches of the Gene Ontology (GO) annotated database for functional categorization (http://www. arabidopsis.org/tools/bulk/go/index.jsp) and of the Pfam database (Pfam 24.0, [33]) to identify the functional domains of the genes.

\section{Verification of absent genes by genotyping}

To confirm the reliability of P/A genes identified, 53 of them were randomly selected for genotyping by PCR. The 
seeds of 80 Arabidopsis accessions (Additional file 4: Table S1) were purchased from the 1001 Genomes Data Center. Twenty-four lines were randomly chosen to verify the absent genes, with $\mathrm{Col}$ as the positive control. For each gene, at least eight lines were chosen to perform a three-primer PCR amplification (two primers were designed in the 3 'and 5 '-flanking regions of the breaking point and one in the insertion of the P/A gene), from which, the present or absent genotype was expected to yield alternative products.

\section{Additional Files}

Additional file 1: Table S2. The genotyping results.

Additional file 2: Table S4. Primer information for the PCR amplification of P/A genes in Table S2.

Additional file 3: Figure S5. The P/A tree and accession tree.

Additional file 4: Table S1. The 80 accessions used in this study.

Additional file 5: Figure S1. Distributions of P/A genes on

chromosomes.

Additional file 6: Table S3. Distributions of P/A genes on

chromosomes.

Additional file 7: Figure S3. GO categories of P/A genes

Additional file 8: Figure S2. Distributions of clustered P/A genes on chromosomes.

Additional file 9: Figure S4. Co-variation between absent allele frequency and nucleotide diversity or gene length.

\section{Competing interests}

The authors declare that they have no competing interests.

\section{Acknowledgements}

The sequence data were produced by the Weigel laboratory at the Max Planck Institute for Developmental Biology. This study was supported by National Natural Science Foundation of China (31071062 \& 30930049), the Fundamental Research Funds for the Central Universities (1084020803 \& 1082020803) and SRFDP to D. T.

\section{Authors' contributions}

DT, SY and ST designed the study. ST contributed extensively to the bioinformatic analyses. ST and YZ performed PCR experiments and $\mathrm{HH}$ assisted the analyses. DT, ST and SY wrote the manuscript. DT and ST prepared and revised the manuscript. All authors read and approved the final manuscript.

\section{Received: 1 September 2011 Accepted: 14 June 2012}

Published: 14 June 2012

\section{References}

1. Morin PA, Luikart G, Wayne RK, Grp SW: SNPs in ecology, evolution and conservation. Trends Ecol Evol 2004, 19(4):208-216.

2. Rafalski A: Applications of single nucleotide polymorphisms in crop genetics. Curr Opin Plant Biol 2002, 5(2):94-100.

3. Sachidanandam R, Weissman D, Schmidt SC, Kakol JM, Stein LD, Marth G, Sherry S, Mullikin JC, Mortimore BJ, Willey DL, et al: A map of human genome sequence variation containing 1.42 million single nucleotide polymorphisms. Nature 2001, 409(6822):928-933.

4. Schmid KJ, Sorensen TR, Stracke R, Torjek O, Altmann T, Mitchell-Olds T, Weisshaar B: Large-scale identification and analysis of genome-wide single-nucleotide polymorphisms for mapping in Arabidopsis thaliana. Genome Res 2003, 13(6A):1250-1257.

5. Grant MR, McDowell JM, Sharpe AG, Zabala MDT, Lydiate DJ, Dangl JL: Independent deletions of a pathogen-resistance gene in Brassica and Arabidopsis. Proc Natl Acad Sci U S A 1998, 95(26):15843-15848.
6. Shen JD, Araki H, Chen LL, Chen JQ, Tian DC: Unique evolutionary mechanism in R-genes under the presence/absence polymorphism in Arabidopsis thaliana. Genetics 2006, 172(2):1243-1250.

7. Henk AD, Warren RF, Innes RW: A new Ac-like transposon of Arabidopsis is associated with a deletion of the RPS5 disease resistance gene. Genetics 1999, 151(4):1581-1589.

8. Morgante M, Brunner S, Pea G, Fengler K, Zuccolo A, Rafalski A: Gene duplication and exon shuffling by helitron-like transposons generate intraspecies diversity in maize. Nat Genet 2005, 37(9):997-1002.

9. Yang $S H$, Feng $Z M$, Zhang $X Y$, Jiang $K$, Jin $X Q$, Hang $Y Y$, Chen JQ, Tian DC: Genome-wide investigation on the genetic variations of rice disease resistance genes. Plant Mol Biol 2006, 62(1-2):181-193.

10. Ding J, Araki H, Wang Q, Zhang P, Yang S, Chen JQ, Tian D: Highly asymmetric rice genomes. BMC Genomics 2007, 8:154

11. lafrate AJ, Feuk T, Van Puymbroeck L, Rivera MN, Listewnik ML, Ying QP, Scherer SW, Lee C: Detection of large-scale variation in the human genome. J Mol Diagn 2004, 6(4):411.

12. Check E: Large genomic differences explain our little quirks. Nature 2005, 435(7040):252-253.

13. Check E: Human genome: patchwork people. Nature 2005, 437 (7062):1084-1086.

14. Kern AD, Begun DJ: Recurrent deletion and gene presence/absence polymorphism: telomere dynamics dominate evolution at the tip of $3 \mathrm{~L}$ in Drosophila melanogaster and D. simulans. Genetics 2008, 179(2):1021-1027

15. Arrach N, Porwollik S, Cheng P, Cho A, Long F, Choi SH, McClelland M: Salmonella serovar identification using PCR-based detection of gene presence and absence. J Clin Microbiol 2008, 46(8):2581-2589.

16. Buckland PR: Polymorphically duplicated genes: their relevance to phenotypic variation in humans. Ann Med 2003, 35(5):308-315.

17. Tuzun E, Sharp AJ, Bailey JA, Kaul R, Morrison VA, Pertz LM, Haugen E, Hayden $\mathrm{H}$, Albertson D, Pinkel D, et al: Fine-scale structural variation of the human genome. Nat Genet 2005, 37(7):727-732.

18. Ha M, Kim ED, Chen ZJ: Duplicate genes increase expression diversity in closely related species and allopolyploids. Proc Natl Acad Sci U S A 2009, 106(7):2295-2300.

19. Fu HH, Dooner HK: Intraspecific violation of genetic colinearity and its implications in maize. Proc Natl Acad Sci U S A 2002, 99(14):9573-9578.

20. Yu J, Wang J, Lin W, Li SG, Li H, Zhou J, Ni PX, Dong W, Hu SN, Zeng CQ, et al: The Genomes of Oryza sativa: a history of duplications. Plos Biol 2005, 3(2):266-281.

21. Adams KL, Wendel JF: Polyploidy and genome evolution in plants. Curr Opin Plant Biol 2005, 8(2):135-141

22. Sebat J, Lakshmi B, Troge J, Alexander J, Young J, Lundin P, Maner S, Massa $\mathrm{H}$, Walker M, Chi MY, et al: Large-scale copy number polymorphism in the human genome. Science 2004, 305(5683):525-528.

23. Ziolkowski PA, Koczyk G, Galganski L, Sadowski J: Genome sequence comparison of $\mathrm{Col}$ and Ler lines reveals the dynamic nature of Arabidopsis chromosomes. Nucleic Acids Res 2009, 37(10):3189-3201.

24. Kaul S, Koo HL, Jenkins J, Rizzo M, Rooney T, Tallon LJ, Feldblyum T, Nierman W, Benito MI, Lin XY, et al: Analysis of the genome sequence of the flowering plant Arabidopsis thaliana. Nature 2000, 408(6814):796-815

25. Tian DC, Araki H, Stahl E, Bergelson J, Kreitman M: Signature of balancing selection in Arabidopsis. Proc Natl Acad Sci U S A 2002, 99(17):11525-11530.

26. Cao J, Schneeberger K, Ossowski S, Gunther T, Bender S, Fitz J, Koenig D, Lanz C, Stegle O, Lippert C, et al: Whole-genome sequencing of multiple Arabidopsis thaliana populations. Nat Genet 2011, 43(10):956-963.

27. Weigel D, Mott R: The 1001 genomes project for Arabidopsis thaliana. Genome Biol 2009, 10(5):107.

28. Schneeberger K, Ossowski S, Ott F, Klein JD, Wang X, Lanz C, Smith LM, Cao J, Fitz J, Warthmann N, et al: Reference-guided assembly of four diverse Arabidopsis thaliana genomes. Proc Natl Acad Sci U S A 2011, 108 (25):10249-10254.

29. Graham GJ: Tandem genes and clustered genes. J Theor Biol 1995, 175 (1):71-87.

30. Round EK, Flowers SK, Richards EJ: Arabidopsis thaliana centromere regions: genetic map positions and repetitive DNA structure. Genome Res 1997, 7(11):1045-1053.

31. Berardini TZ, Mundodi S, Reiser L, Huala E, Garcia-Hernandez M, Zhang PF, Mueller LA, Yoon J, Doyle A, Lander G, et al: Functional annotation of the 
Arabidopsis genome using controlled vocabularies. Plant Physiol 2004, 135(2):745-755.

32. Consortium GO: Creating the gene ontology resource: design and implementation. Genome Res 2001, 11(8):1425-1433.

33. Finn RD, Mistry J, Tate J, Coggill P, Heger A, Pollington JE, Gavin OL, Gunasekaran P, Ceric G, Forslund K, et al: The Pfam protein families database. Nucleic Acids Res 2010, 38(Database issue):D211-D222.

34. Zhang XC, Wang Z, Zhang X, Le MH, Sun J, Xu D, Cheng J, Stacey G: Evolutionary dynamics of protein domain architecture in plants. $B M C$ Evol Biol 2012, 12(1):6.

35. Kersting AR, Bauer EB, Moore AD, Grath S: Dynamics and adaptive benefits of protein domain emergence and arrangements during plant genome evolution. Genome Biol Evol 2012, 4(3):316-329.

36. Ellis JG, Lawrence GJ, Luck JE, Dodds PN: Identification of regions in alleles of the flax rust resistance gene $L$ that determine differences in gene-for-gene specificity. Plant Cell 1999, 11(3):495-506.

37. Bakker EG, Toomajian C, Kreitman M, Bergelson J: A genome-wide survey of R gene polymorphisms in Arabidopsis. Plant Cell 2006, 18(8):1803-1818.

38. Clark RM, Schweikert G, Toomajian C, Ossowski S, Zeller G, Shinn P, Warthmann N, Hu TT, Fu G, Hinds DA, et al: Common sequence polymorphisms shaping genetic diversity in Arabidopsis thaliana. Science 2007, 317(5836):338-342

39. Thomas $\mathrm{JH}$ : Adaptive evolution in two large families of ubiquitin-ligase adapters in nematodes and plants. Genome Res 2006, 16(8):1017-1030.

40. Zhang QF, Maroof MAS, Lu TY, Shen BZ: Genetic diversity and differentiation of Indica and Japonica rice detected by Rflp analysis. Theor Appl Genet 1992, 83(4):495-499.

41. Copenhaver GP, Nickel K, Kuromori T, Benito Ml, Kaul S, Lin X, Bevan M, Murphy G, Harris B, Parnell LD, et al: Genetic definition and sequence analysis of Arabidopsis centromeres. Science 1999, 286(5449):2468-2474.

42. Lee HR, Zhang WL, Langdon T, Jin WW, Yan HH, Cheng ZK, Jiang JM: Chromatin immunoprecipitation cloning reveals rapid evolutionary patterns of centromeric DNA in Oryza species. Proc Natl Acad Sci U S A 2005, 102(33):11793-11798.

43. Murphy WJ, Larkin DM, Everts-van der Wind A, Bourque G, Tesler G, Auvil L, Beever JE, Chowdhary BP, Galibert F, Gatzke L, et al: Dynamics of mammalian chromosome evolution inferred from multispecies comparative maps. Science 2005, 309(5734):613-617.

44. Henikoff S, Ahmad K, Malik HS: The centromere paradox: stable inheritance with rapidly evolving DNA. Science 2001, 293(5532):1098-1102.

45. Charlesworth B, Sniegowski P, Stephan W: The evolutionary dynamics of repetitive DNA in eukaryotes. Nature 1994, 371(6494):215-220.

46. Shi J, Wolf SE, Burke JM, Presting GG, Ross-Ibarra J, Dawe RK: Widespread gene conversion in centromere cores. Plos Biol 2010, 8(3):e1000327.

47. Ohno S: Evolution by Gene Duplication. Berlin, New York: Springer; 1970

48. Van der Hoorn RA, De Wit PJ, Joosten MH: Balancing selection favors guarding resistance proteins. Trends Plant Sci 2002, 7(2):67-71.

49. Yamada K, Lim J, Dale JM, Chen H, Shinn P, Palm CJ, Southwick AM, Wu HC, Kim C, Nguyen M, et al: Empirical analysis of transcriptional activity in the Arabidopsis genome. Science 2003, 302(5646):842-846.

50. Wilgenbusch JC, Swofford D: Inferring evolutionary trees with PAUP*. Curr Protoc Bioinforma 2003, Chapter 6:Unit 64.

51. Tamura K, Peterson D, Peterson N, Stecher G, Nei M, Kumar S: MEGA5: Molecular Evolutionary Genetics Analysis using maximum likelihood, evolutionary distance, and maximum parsimony methods. Mol Biol Evol 2011, 28 (10):2731-2739.

52. Sennblad B, Schreil E, Berglund Sonnhammer AC, Lagergren J, Arvestad L: Primetv: a viewer for reconciled trees. BMC Bioinforma 2007, 8:148.

53. Richly E, Kurth J, Leister D: Mode of amplification and reorganization of resistance genes during recent Arabidopsis thaliana evolution. Mol Biol Evol 2002, 19(1):76-84

doi:10.1186/1471-2148-12-86

Cite this article as: Tan et al:: Variation of presence/absence genes among Arabidopsis populations. BMC Evolutionary Biology 2012 12:86

\section{Submit your next manuscript to BioMed Central and take full advantage of:}

- Convenient online submission

- Thorough peer review

- No space constraints or color figure charges

- Immediate publication on acceptance

- Inclusion in PubMed, CAS, Scopus and Google Scholar

- Research which is freely available for redistribution 